\title{
The star formation history of the Universe as revealed by deep radio observations
}

\author{
N. Seymour, ${ }^{1 \star}$ T. Dwelly, ${ }^{2}$ D. Moss, ${ }^{2}$ I. McHardy, ${ }^{2}$ A. Zoghbi, ${ }^{2,3}$ G. Rieke, ${ }^{4}$ M. Page, ${ }^{5}$ \\ A. Hopkins ${ }^{6}$ and N. Loaring ${ }^{7}$ \\ ${ }^{1}$ Spitzer Science Center, Caltech, 1200 East California Boulevard, Pasadena, CA 91125, USA \\ ${ }^{2}$ School of Astronomy \& Astrophysics, University of Southampton, Highfield, Southampton SO17 1BJ \\ ${ }^{3}$ Institute of Astronomy, University of Cambridge, Madingley Road, Cambridge CB3 OHA \\ ${ }^{4}$ Steward Observatory, Tucson, USA \\ ${ }^{5}$ Mullard Space Science Laboratory, UCL, Holmbury St Mary, Dorking, Surrey RH5 6NT \\ ${ }^{6}$ University of Sydney, Australia \\ ${ }^{7}$ SALT, PO box 9, Observatory 7925, South Africa
}

Accepted 2008 February 24. Received 2008 February 20; in original form 2007 October 29

\begin{abstract}
Discerning the exact nature of the sub-mJy radio population has been historically difficult due to the low luminosity of these sources at most wavelengths. Using deep ground based optical follow-up and observations from the Spitzer Space Telescope we are able to disentangle the radio-selected active galactic nuclei (AGN) and star-forming galaxy (SFG) populations for the first time in a deep multifrequency VLA/MERLIN Survey of the $13^{\mathrm{H}} X M M-$ Newton/Chandra Deep Field. The discrimination diagnostics include radio morphology, radio spectral index, radio/near-infrared (near-IR) and mid-IR/radio flux density ratios. We are now able to calculate the extragalactic Euclidean normalized source counts separately for AGN and SFGs. We find that while SFGs dominate at the faintest flux densities and account for the majority of the upturn in the counts, AGN still make up around one quarter of the counts at $\sim 50 \mu \mathrm{Jy}(1.4 \mathrm{GHz})$. Using radio luminosity as an unobscured star formation rate (SFR) measure we are then able to examine the comoving SFR density of the Universe up to $z=3$ which agrees well with measures at other wavelengths. We find a rough correlation of SFR with stellar mass for both the sample presented here and a sample of local radio-selected SFGs from the 6df-NVSS survey. This work also confirms the existence of, and provides alternative evidence for, the evolution of distribution of star formation by galaxy mass: 'downsizing'. As both these samples are SFR-selected, this result suggests that there is a maximum SFR for a given galaxy that depends linearly on its stellar mass. The low 'characteristic times' (inverse specific SFR) of the SFGs in our sample are similar to those of the 6dF-NVSS sample, implying that most of these sources are in a current phase of enhanced star formation.
\end{abstract}

Key words: galaxies: evolution - galaxies: starburst - radio continuum: galaxies.

\section{INTRODUCTION}

Whilst observations of powerful, radio-loud active galactic nuclei (AGN) were an early probe of the distant Universe (see Stern \& Spinrad 1999, for a summary), starburst galaxies are three or more orders of magnitudes less luminous at radio wavelengths and hence difficult to observe at large distances. However, the deepest radio surveys at $1.4 \mathrm{GHz}$ now reach an rms below $10 \mu \mathrm{Jy}$ (Richards 2000; Seymour, McHardy \& Gunn 2004; Biggs \& Ivison 2006; Fomalont et al. 2006). These deep radio observations reveal a (very well-

^E-mail: seymour@ipac.caltech.edu characterized) upturn in the Euclidean normalized sources counts below $1 \mathrm{mJy}$ above that predicted from the extrapolation of the AGN counts measured at brighter flux densities. This upturn has been attributed to the emergence of a star-forming galaxy (SFG) population, requiring strong evolution of the SFG radio luminosity function(LF) (Rowan-Robinson et al. 1993; Hopkins et al. 1998; Seymour et al. 2004; Moss et al. 2007), although some authors argue that there is a significant contribution due to relatively weak radio AGN (Simpson et al. 2006; Barger, Cowie \& Wang 2007; Huynh, Jackson \& Norris 2007).

Determining the nature of individual radio sources has remained difficult due to their low luminosities at other wavelengths. Discrimination between radio-selected SFGs and AGN in these deep 
surveys would, for example, allow an independent measure of the star formation history of the Universe as star formation rate (SFR) is directly related to radio luminosity for galaxies with weak or no AGN radio emission (Condon 1992). The comoving SFR density (SFRD) has been determined previously from deep radio data out to $z \sim 1.6$ (Haarsma et al. 2000), but the study presented here offers several improvements and advantages, noticeably a more robust and systematic discrimination between AGN and SFG, the use of more accurate redshifts, an extension to higher redshift and a larger area/sample size by a factor of $\sim 5$.

Whilst radio surveys have not traditionally been used to determine SFRs in the distant Universe, other methods have their limitations. The far-infrared (far-IR) and submillimetre wavelength range is limited by the relatively poor sensitivity and angular resolution of current instrumentation and telescopes. The mid-IR, whilst very sensitive, is not as reliable a tracer of SFR at high redshift as the far-IR and submillimetre (e.g. Papovich et al. 2007). Ultraviolet (UV) emission from young massive stars can easily be obscured by dust, but the extrapolation of models derived from local galaxies likely break down for the more luminous galaxies, $>L_{*}$, found in deep optical surveys. Furthermore, the rest-frame UV is redshifted across a large wavelength range (UV/optical/near-IR) at cosmological distances so different detectors are needed to trace the UV at different redshifts. Determination of the SFR from optical emission lines is also affected by obscuration and shifting to longer wavelengths and hence in and out of observable wavelength windows. The optical emission lines can also suffer from absorption by dust. $\mathrm{X}$-ray emission associated with star formation (e.g. from binary stars) is intrinsically weak and is subject to varying degrees of photoelectric absorption. Even at the faintest $\mathrm{X}$-ray fluxes reached in the Chandra Deep Field-North (CDF-N) $\left(\sim 10^{-17} \mathrm{erg} \mathrm{cm}^{-2} \mathrm{~s}^{-1}\right)$, the source counts are dominated by AGN (Bauer et al. 2004). Given that forthcoming radio facilities (e.g. eVLA, eMERLIN, LOFAR, ASKAP and eventually the SKA) will reach many orders of magnitude deeper than the current deepest radio survey, and will be able to detect SFGs out to the era of re-ionization $(z \lesssim 7)$, it is timely to consider how to characterize the emission from faint radio sources and which supporting data are the most valuable.

We make an important distinction here between radio indicators of an AGN and non-radio indicators of an AGN. We need to separate the radio sources into those whose radio emission is AGN dominated and those that are consistent with being dominated by star formation. By AGN powered indicators we mean some measure that indicates that the radio emission from a galaxy is dominated by accretion on to a supermassive black hole and/or from associated jets and lobes (i.e. radio-loud AGN). We assume, for simplicity, that the dominant power in most sources is either from an AGN or from star formation, but otherwise make no a priori assumptions about the sources, e.g. the spectral energy distribution (SED). The radio AGN indicators we use in this paper are radio/near-IR flux density ratio, mid-IR/radio flux density ratio, radio morphology and radio spectral index. We do not include non-radio AGN indicators, e.g. classical methods like optical/IR emission lines, optical morphology, X-ray observations, etc. and methods from recent results with the Spitzer Space Telescope based on mid-IR SEDs (e.g. Donley et al. 2005; Lacy et al. 2005; Stern et al. 2005). As these non-radio indicators tell us nothing directly about the nature of the radio emission we do not use them in our discrimination methods. A comparable philosophy was previously adopted by Muxlow et al. (2005).

In this paper we will present our method for classifying the faint radio sources and examine the properties of the SFG population. The radio, optical and IR observations are summarized in Section 2.
The various methods of discriminating between AGN and SFGs are presented in Section 3. In Section 4 we present the Euclidean normalized source counts separately for AGN and SFGs for the first time. In Section 5 we study the general properties of the radio-selected SFG population, present the comoving SFRD of the Universe up to redshift $\sim 3$ derived from our data, examine the distribution of star formation with host galaxy stellar mass and derive characteristic times. We conclude this article in Section 6. Throughout we use a concordance model of Universe expansion, $\Omega_{\mathrm{M}}=1-\Omega_{\Lambda}=0.3$, $\Omega_{0}=1$ and $H_{0}=70 \mathrm{~km} \mathrm{~s}^{-1} \mathrm{Mpc}^{-1}$ (Spergel et al. 2003). Magnitudes are $\mathrm{AB}$ unless otherwise stated.

\section{THE DATA}

The $13^{\mathrm{H}}$ XMM-Newton/Chandra Deep Field Survey presents us with a unique data set for performing a radio-based SFG/AGN separation of radio sources. This field, centred at $13^{\mathrm{h}} 34^{\mathrm{m}} 37^{\mathrm{s}}+$ $37^{\circ} 54^{\prime} 44^{\prime \prime}$, was the location of one of the deepest ROSAT surveys (McHardy et al. 1998), and lies in a region of extremely low Galactic absorption $\left(N_{\mathrm{H}} \sim 6 \times 10^{19} \mathrm{~cm}^{-2}\right)$.

\subsection{The radio data}

\subsubsection{The VLA 1.4-GHz observations}

The $13^{\mathrm{H}}$ XMM-Newton/CDF was observed by the Very Large Array (VLA) at $1.4 \mathrm{GHz}$ for $14 \mathrm{~h}$ in A-array in 1996 August and $10 \mathrm{~h}$ in Barray in 1995 November. A catalogue of 449 sources above $30 \mu \mathrm{Jy}$ ( $4 \sigma$ detection limit) in a 30 arcmin diameter circular field of view $\left(0.196 \mathrm{deg}^{2}\right)$ was obtained with a resolution of $3.3 \mathrm{arcsec}$. The Euclidean normalized source counts, corrected for the incompleteness of the catalogue due to instrumental effects, were presented in Seymour et al. (2004, hereafter S04). The 449 sources detected at $1.4 \mathrm{GHz}$ with the VLA constitute the parent sample on which this work is based. In contrast to S04 we use the peak flux density instead of total flux density for sources that are significantly resolved and at low signal-to-noise ratio $(\mathrm{S} / \mathrm{N})$ as the peak flux is a better measure of the true flux in these cases.

The size of this sample (in terms of area and number of sources) is well positioned between those fields of the similar area but are slightly deeper [by $\sim 40$ per cent, e.g. the Lockman Hole and the Hubble Deep Field-North (HDF-N); Biggs \& Ivison 2006] and those of larger area (e.g. AEGIS and COSMOS; Ivison et al. 2007a; Schinnerer et al. 2007), but which are less deep by $\sim 40$ per cent.

\subsubsection{The MERLIN 1.4-GHz observations}

The $13^{\mathrm{H}}$ field was observed with Multi-Element Radio-LInked Network (MERLIN) in 1999 April. Due to the smaller field of view, 10 arcmin, four MERLIN pointings were used to cover most of the VLA field of view. MERLIN has a higher resolution, 0.2 arcsec, than the VLA due to its greater maximum baseline. We used the parent catalogue as a detection list and only imaged the positions around known sources (including bright sources not in the principle VLA or MERLIN field of view). We made MERLIN images for all VLA detections with $S_{1.4 \mathrm{GHz}}>72 \mu \mathrm{Jy}$ that lay inside the MERLIN coverage. These images were combined by first making maps without the beam deconvolved and beams around each source in the sky plane, then averaging the maps and beams before deconvolving the average beam from the average map as described by Zoghbi et al. (in preparation) The combination of data sets from different telescope arrays is non-trivial as the four MERLIN pointings are 
offset from the VLA phase centre and both the VLA and MERLIN images suffer from radial smearing. However, for sources in the parent sample having flux densities above $\sim 100 \mu \mathrm{Jy}$, and that are favourably positioned, we can make high-resolution maps from combined MERLIN and VLA data.

\subsubsection{The VLA 4.8-GHz observations}

The $13^{\mathrm{H}}$ field was observed with the VLA at $4.8 \mathrm{GHz}$ in 1991 April. The observations cover the whole field of view of the 1.4-GHz data, 30 arcmin (observations originally designed to match the ROSAT field of view McHardy et al. 1998) with 51 separate pointings. The observations reach $33 \mu \mathrm{Jy} \mathrm{rms}$, and were performed in VLA D-array hence the restoring beam is 14 arcsec, around a factor of 4 greater than the 1.4-GHz observations. From the catalogue of Seymour (2002) we find 45 sources with flux densities $>4 \sigma$ and within the 30 arcmin diameter field of view of the $1.4-\mathrm{GHz}$ observations. Of these sources 45 have counterparts in the $1.4-\mathrm{GHz}$ catalogue within 7 arcsec and hence we can calculate their $1.4-4.8 \mathrm{GHz}$ spectral indices. We define the radio spectral index, $\alpha$, by $S_{v} \propto v^{\alpha}$, and hence $\alpha=-1.85 \log _{10}\left(S_{1.4} / S_{4.86}\right)$. We use total, rather than peak, flux densities when calculating the radio spectral index. In our earlier study (S04), we made tapered maps of the 1.4-GHz data but we did not find significant extended radio emission that could affect the calculated spectral indices. There is one instance where two nearby 1.4-GHz sources (separated by 20 arcsec) are matched to a single 4.8-GHz source and therefore we cannot calculate the spectral indices separately for these two sources.

\subsection{Optical, near-IR and mid-IR data}

\subsubsection{Optical and near-IR data}

We have obtained imaging in many bands from the near-UV to nearIR over recent years: $u^{*}, g^{\prime}$ and $i^{\prime}$ band from MegaCam/CanadaFrance-Hawaii Telescope (CFHT), B,R,I and $z^{\prime}$ band from SuprimeCam/Subaru, $Z$ band from WFC/INT, $J$ band from WIRC/Palomar, $H$ band from WIRCam/CFHT and $K$ band from WFCAM/UKIRT. This imaging covers the entire parent sample bar the WIRC $J$ band (which covers approximately three-fourth of the survey area), and has been supplemented by hundreds of optical spectra of the counterparts to our radio and X-ray sources. Of our parent sample 164/449 currently have redshifts determined from optical spectroscopy.

\subsubsection{Mid-IR data}

The $13^{\mathrm{H}}$ field was observed by the IRAC (Fazio et al. 2004) and MIPS instruments (Rieke et al. 2004) onboard the Spitzer Space Telescope (Werner et al. 2004) in 2005 July as part of MIPS instrument team GTO time (PI G. Rieke, programme identification number 81 ). A strip of $\sim 0.5 \times 1 \mathrm{deg}^{2}$ containing the $13^{\mathrm{H}}$ field was imaged by both instruments. The IRAC data consisted of observations with all four channels $(3.6,4.5,5.6$ and $8.0 \mu \mathrm{m})$ reaching $1 \sigma$ flux density limits of $0.7,1.2,7.2$ and $7.6 \mu \mathrm{Jy}$, respectively. These values represent the limiting flux density of a point source for which we can make a flux measurement in a 3.8 arcsec diameter aperture with $>1 \sigma$ accuracy. Most, 388/449 (87 per cent), of our parent sample were detected in at least the $3.6 \mu \mathrm{m}$ channel. The $24 \mu \mathrm{m}$ data were taken as part of a simultaneous scan map in each of the three bands $(24,70$ and $160 \mu \mathrm{m})$ reaching a $3 \sigma$ detection limit of $98 \mu \mathrm{Jy}$ in a 10.5-arcsec aperture. We found 330/449 (73 per cent) of our parent sample had 24- $\mu \mathrm{m}$ counterparts. We do not use the 70 and $160 \mu \mathrm{m}$ data here as they are not sensitive enough to provide information on most of the radio sources, and then only those at low redshift.

\subsubsection{Photometric redshifts}

We have used our multiband near-UV to near-IR together with IRAC 3.6, 4.5 and $5.8 \mu \mathrm{m}$ data to calculate photometric redshifts for the optical counterparts to our radio sources. Full details of this process will be presented in Dwelly et al. (in preparation), but here we give a short summary. Initial catalogues were generated independently for each optical, near-IR and mid-IR waveband using SEXTRACTOR (Bertin \& Arnouts 1996). These initial catalogues are then combined into a single 'master' catalogue containing only unique detections. Aperture photometry is carried out in each waveband at the locations of each master catalogue source. In the optical/nearIR bands we used a 3 arcsec diameter aperture for bright sources $\left(i^{\prime}<20\right)$, and a 2 -arcsec aperture for fainter sources to improve $\mathrm{S} / \mathrm{N}$. In the IRAC bands a 5.8 arcsec diameter aperture was employed. Appropriate aperture corrections are applied given the different point spread functions in each waveband (Gawiser et al. 2006). We match optical/near-IR counterparts to the parent radio sample are by searching for all objects in the 'master' catalogue lying within $1.5 \mathrm{arcsec}$ of the peak of the radio emission. Where available we use the high-resolution images from combined MERLIN + VLA maps to determine the correct optical counterparts. We examine the optical and radio images for sources with more than one counterpart, and manually choose the most appropriate object. We then used the publicly available HYPERZ code (Bolzonella, Miralles \& Pelló 2000) to determine photometric redshifts for each source from their multiband aperture photometry. Only the wavebands shortward of $5.8 \mu \mathrm{m}$ were used to determine photometric redshifts in order to avoid complications with polycyclic aromatic hydrocarbon (PAH) features. We used the standard set of synthetic evolving galaxy templates supplied with HYPERZ. To ensure reliability, we ignore the photometric redshifts calculated for all radio sources detected with $>3 \sigma$ significance in fewer than four wavebands. Hence, 60 radio sources from our parent sample have no photometric redshift information including 31 with no counterparts detected in any optical, near-IR or mid-IR waveband. Most of the other 29 sources are detected only in the $K$-band data and/or the two shorter wavelength IRAC channels, and hence the derived photometric redshifts have very large uncertainties. We consider the likely nature of these objects in Section 3.6.1.

\subsection{Local 6dF-NVSS comparison sample}

To illustrate our discrimination techniques we use the 6dF NRAO VLA Sky Survey (6dF-NVSS) sample of local spectroscopically identified radio sources from Mauch \& Sadler (2007). These authors identified $\sim 8000$ radio sources from the $1.4-\mathrm{GHz}$ NVSS (Condon 1992) in the Second Incremental Data Release of the 6dF Galaxy Survey (6dFGSDR2) with galaxies brighter than $K=12.75$ (Vega) from the 2MASS Extended Source Catalogue. This sample covers about 17 per cent of the sky and includes galaxies across $0.003<z<$ 0.3. Mauch \& Sadler (2007) discriminated between AGN and SFGs using emission and absorption features in the $6 \mathrm{dF}$ spectra and derived local LFs for both populations using the Saunders et al. (1990) form of the LF which is commonly used at long wavelengths (i.e. mid-IR and longward). Their sample has also been cross-matched 
with the IRAS Faint Source Catalogue providing fluxes at 12, 25, 60 and $100 \mu \mathrm{m}$ which they use to confirm the radio-IR correlation. The Mauch \& Sadler (2007) catalogue provides us with a local reference sample of SFGs with known radio, IR and $K$-band flux densities (from which we can derive SFRs and stellar masses) to which we can compare our results from higher redshift.

\section{RADIO-BASED EMISSION DIAGNOSTICS}

Here we present four radio emission diagnostics which directly probe the physical origin of the radio luminosity: two of these are purely from the radio data (radio spectral indices, morphology), and two involve comparisons with observed flux densities at other wavelengths (mid-IR and near-IR) as a function of redshift. These diagnostics are mostly positive AGN discriminators, i.e. they generally imply that the radio emission of a source is very likely powered by AGN emission and not star formation. We do not include the obvious radio luminosity discriminator, where a radio luminosity $>$ $10^{25} \mathrm{~W} \mathrm{~Hz}^{-1}$ would imply an unphysical SFR of $\sim 5000 \mathrm{M}_{\odot} \mathrm{yr}^{-1}$, as those eight sources clearly above this luminosity are flagged as AGN by one or more of the other methods below and potentially such objects do exist in small numbers at high redshift.

We give the number of sources from our parent sample for which each of our AGN/SFG discriminators is applicable in Table 1 along with a short comment of why they apply to only such a number. We discuss the implications of these selection functions in Section 3.5.

\subsection{Radio morphology}

Only six sources from the parent sample show the classical unambiguous AGN signature of a double lobed, FR2 morphology (i.e. Fanaroff \& Riley 1974) in our 3.3-arcsec resolution imaging. For other extended VLA sources there is not enough detail to distinguish between a clear AGN morphology and extended, galaxy scale emission from star formation.

Additionally we include results of the combined MERLIN + VLA (A-array) maps at $1.4 \mathrm{GHz}$ (Zoghbi et al., in preparation) which have a resolution of 0.5 arcsec. These results are limited to the brighter objects from our master sample due to the sensitivity of our MERLIN images, and also the inner $\sim 20$ arcmin covered by the four MERLIN pointings. We are therefore able to make combined maps for 127 sources. Many show clear AGN jet/lobe morphology including the six sources flagged as AGN from the lower resolution VLA images. The other sources show either a very compact, unresolved morphology or are extended on $\sim 1$-arcsec scales, often almost completely resolved out due to the lack of sensitivity of MERLIN to extended structure. Although the unresolved sources do not have significantly constrained brightness temperatures $\left(\geqslant 10^{3} \mathrm{~K}\right)$ this compactness is most likely due to an AGN (e.g. Muxlow et al. 2005), but a small chance remains that they could be due to nuclear starbursts. The sources with extended emission over the galaxy

Table 1. Number of sources from our parent sample for which each SFG/AGN discrimination method is applicable. We list discrimination method, the number of sources which it is applicable to and the reason why.

\begin{tabular}{lcc}
\hline Discrimination & $\#$ & Limitation of method \\
\hline Morphology & 127 & Brightest sources only and coverage \\
Spectral index & 45 & Brightest sources only \\
$S_{24 \mu \mathrm{m}} / S_{1.4 \mathrm{GHz}}$ & 445 & Limited by $24-\mu \mathrm{m}$ coverage \\
$S_{1.4 \mathrm{GHz}} / S_{2.2 \mu \mathrm{m}}$ & 449 & Applies to all sources \\
\hline
\end{tabular}

which is often completely resolved out must be from star formation occurring throughout the galaxy. Hence, the MERLIN + VLA images show that $42 / 127$ sources are very likely to be AGN.

\subsection{Radio spectral indices}

Using our 4.8-GHz data (Seymour 2002) we have radio spectral indices for 45 sources in our sample. These are among the brightest sources in our sample as the $4.8 \mathrm{GHz}$ rms of $30 \mu \mathrm{Jy}$ corresponds to $\sim 70 \mu \mathrm{Jy}$ at $1.4 \mathrm{GHz}$ assuming $\alpha_{1.4}^{4.8}=-0.8$. Radio emission from star formation, due to synchrotron radiation, will generally have a spectrum with a slope in the range $-0.5 \lesssim \alpha_{1.4}^{4.8} \lesssim-1.0$ (Thompson et al. 2006). Radio sources with spectral indices steeper than $\alpha=$ -1 are typically targeted as high-redshift radio galaxies. Therefore any radio sources with spectral indices not in the range $-0.4 \lesssim$ $\alpha_{1.4}^{4.8} \lesssim-1.1$ (allowing for the errors in the flux densities) we flag as having AGN powered radio emission. We find 31 sources have spectral indices not in this range (i.e. with $\alpha_{1.4}^{4.8} \geqslant-0.4$ or $\alpha_{1.4}^{4.8} \leqslant$ $-1.1)$.

This diagnostic is particularly powerful as it is relatively insensitive to redshift; e.g. Klamer et al. (2006) find that 90 per cent of a sample of high-redshift AGN have radio spectra best characterized as a power law with no spectral steepening. It is possible that high-redshift SFGs could have flatter radio spectra due to free-free processes dominating the higher rest-frame frequencies, especially if the source is very young. However, the $1.4-\mathrm{GHz}$ counterparts to the $4.8-\mathrm{GHz}$ sources are typically bright ( $>1 \mathrm{mJy}$ at $1.4 \mathrm{GHz}$ ) hence unlikely to include many high-redshift SFGs. Most radio sources classified as AGN by their radio spectral index are also classified by other diagnostics (see Table 3).

\subsection{Radio to near-IR flux density ratios}

'Radio-loudness' is another classical discriminator of radio luminous AGN (Kellerman et al. 1989) and traditionally based on radio to optical (observed or rest-frame) flux density ratio. More recently radio-loudness has been defined in terms of absolute radio luminosity (e.g. $L_{1.4 \mathrm{GHz}} \gtrsim 10^{25} \mathrm{~W} \mathrm{~Hz}^{-1}$ Miller, Peacock \& Mead 1990 , but this diagnostic would only apply to eight of our parent sample and those have already been flagged as an AGN by one or both of the previous two diagnostics. The original definition of radio-loudness was based on $B$-band observations, but given that at the redshifts in question, $0 \leqslant z \leqslant 4$, this band quickly starts sampling below the 4000- $\AA$ break where variations in SFR and absorption by dust have a strong effect, we chose to use a longer wavelength band. In fact the only band shortward of the mid-IR which remains longward of the 4000 - $\AA$ break up to $z \sim 4$ is $K$ band. Our $K$-band data are particularly deep: $K=22.6$ ( $3 \sigma$ limit).

In keeping with our philosophy stated in Section 1, where we make no assumptions about the observed SED, we plot the observed flux density ratios against redshift. Given that we do not know the nature of each radio source beforehand, we can only plot the un$k$-corrected flux density ratios and compare them with tracks of different AGN and starburst galaxies. This approach avoids making any a priori assumptions about the nature of each source. In Fig. 1(a) we present the $1.4 \mathrm{GHz}$ to $K$-band flux density ratio against redshift. We plot the tracks of two SFGs which have no strong AGN component. These are luminous infrared galaxy (LIRG) and ultraluminous infrared galaxy (ULIRG) templates made from average composites from fits to about a dozen LIRGs and ULIRGs with very high fidelity observations. From 5-35 $\mu \mathrm{m}$ they are based on Spitzer IRS long low spectra. For $1-5 \mu \mathrm{m}$, the templates are built from stellar population models, with overall slopes constrained by large beam IRAC and 

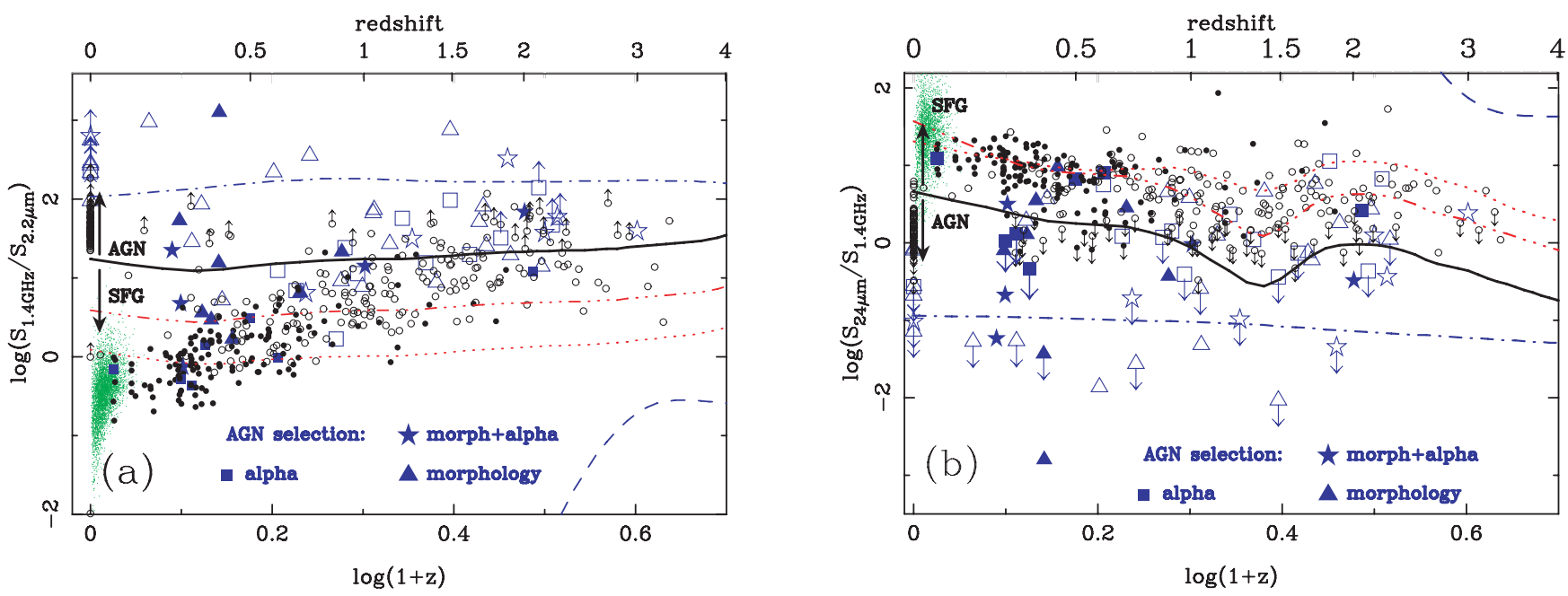

Figure 1. (a) $1.4 \mathrm{GHz}$ to near-IR flux density ratio plotted against redshift and (b) $24 \mu \mathrm{m}$ to $1.4-\mathrm{GHz}$ flux density ratio plotted against redshift. Filled symbols have spectroscopic redshifts and open symbols have photometric redshifts. The large blue symbols are those radio sources confirmed as AGN from their morphology (triangles), spectral index (squares) or both (stars). We show the location of the flux density ratios of the local 6dF-NVSS sample with green dots. The tracks of SFGs with luminosities over the range of interest are marked: LIRG (red dotted lines), ULIRG (red dot-dashed lines). See text more discussion of the templates used. We also overplot the tracks of a radio-loud (blue dash-dot-dot-dotted lines) and a radio-quiet QSO (blue dashed lines) from Elvis et al. (1994). The cut-off (solid black lines) in both cases is chosen to be $0.6 \mathrm{dex}(=5 \sigma)$ above or below the more extreme of two the SFG tracks. Sources without known redshifts are plotted at $z=0$.

2MASS photometry. The radio regime was determined with data from Condon et al. (1991). The corresponding SFRs of these two templates are approximately $\sim 10$ and $\sim 100 \mathrm{M}_{\odot} \mathrm{yr}^{-1}$, respectively. We also plot the tracks of classical 'radio-loud' and 'radio-quiet' QSOs from the templates of Elvis et al. (1994).

Fig. 1(a) shows several clear radio-loud AGN with flux density ratios similar to or greater than the radio-loud QSO template track. We can use this plot to define a locus where a radio source will unambiguously identified as an AGN. We decide to take a cut $5 \sigma$ above the higher SFG track (the ULIRG one) where $\sigma$ comes from taking an uncertainty of 20 per cent in the radio flux density and 20 per cent in the $K$-band magnitude. We emphasize that these uncertainties are extremely conservative and that the majority of radio sources will have much smaller uncertainties, but we are also allowing for the uncertainty in the SEDs used for the template tracks. Combining these uncertainties we get a $5 \sigma$ cut of $0.6 \mathrm{dex}$ $\left[=5 \sqrt{(}\left(0.2^{2}+0.2^{2}\right) / \ln (10)\right]$.

We note that all bar one of the sources with unknown redshifts are well above our AGN/SFG discrimination line at almost all redshifts and hence are most likely all obscured AGN at high redshift (see Section 3.5.1 for further discussion of these sources).

\subsection{Radio to mid-IR flux density ratios}

The correlation of the radio and far-IR luminosities of SFGs is well established over five orders of magnitude (Yun, Reddy \& Condon 2001) and is one of the tightest results in astrophysics. The continuation of this relationship in the mid-IR and to $z \geqslant 1$ is also fairly well established (Garrett 2002; Appleton et al. 2004). Furthermore we show in Appendix A that the total IR to radio luminosity relationship does continue to high redshifts for ULIRGs. This relationship can be used as another clear discriminant of radio-loud AGN; all objects with a relative 'radio-excess' are very likely to be AGN.

Spitzer provides comparably deep IR observations to the radio which allows comparison of the IR properties of the sub-mJy radio population. We choose to use the $24 \mu \mathrm{m}$ band of the MIPS instrument as it is more sensitive than the $70 \mu \mathrm{m}$ band with a $3 \sigma$ detection limit of $98 \mu \mathrm{Jy}$ compared to $3 \sigma \sim 3 \mathrm{mJy}$ at $70 \mu \mathrm{m}$. This
$24 \mu \mathrm{m}$ detection limit should be sufficient to detect all the luminous starburst galaxies $\left(L_{24 \mu \mathrm{m}} \gtrsim 3 \times 10^{10} \mathrm{~L}_{\odot}\right)$ which we could detect at radio wavelengths out to $z \sim 1$ and most starburst galaxies at $z>1$, given the nominal $1.4-\mathrm{GHz}$ detection limit of our parent sample.

In Fig. 1(b) we plot the distribution of mid-IR to radio flux density ratios, $S_{24 \mu \mathrm{m}} / S_{1.4 \mathrm{GHz}}$, against redshift for sources in our sample. We compare this distribution to redshifted starburst and AGN templates tracks in a similar way to Section 3.3. On the whole a large number of $24 \mu \mathrm{m}$ detected sources follow the SFG template tracks suggesting that SFGs make up a significant fraction of the submJy radio population. There are many obvious 'radio-excess' and a few 'IR-excess' sources which are both likely to harbour AGN. The radio-excess sources are likely to host AGN due to their radio-loud nature. The IR-excess sources are likely to host radio-quiet AGN with hot dust dominating in the mid-IR, i.e. radio-quiet obscured AGN such as those found by Lacy et al. (2007), but with moderate amounts of star formation dominating the radio emission. These 'IR-excess' sources likely explain the high the mid-IR to radio flux density ratio seen in Boyle et al. (2007) due to the selection effects of that study. The general trend toward lower mid-IR ratios at higher redshifts and the starburst SEDs presented suggest that the low midIR ratio found by Beswick et al. (2008) is due to $k$-correction effects to the observed ratio.

As in the previous section we use a $5 \sigma$ cut-off below the more extreme starburst. We again get a cut-off value of $5 \sigma=0.6 \mathrm{dex}$ from assuming a median 20 per cent uncertainty in both the $1.4 \mathrm{GHz}$ and $24 \mu \mathrm{m}$ flux density. This cut also allows for uncertainty in the SED tracks.

\subsection{Results of AGN/SFG discrimination}

Using the above four discriminators we can discriminate the AGN from the SFGs on a statistical basis. Although most of these discriminators are mainly just positive AGN identifiers we can be reasonably confident that we have removed nearly all the AGN, at least statistically. In fact, 58 per cent of the sources flagged as AGN are done so by two or more discriminators. We find that $178 / 449$ sources 
show clear indications that their radio emission is due to AGN activity. One radio source is, surprisingly, identified as a star from optical spectroscopy. These results are summarized in Table 2, along with the incompleteness corrected number and percentage. This incompleteness factor is a correction for the decrease in the sky area probed at faint fluxes due to the decrease in sensitivity of our radio map away from the pointing centre.

We look at the break down of radio AGN classification in Table 3. We find that the two most effective methods are the flux density ratios which each account for $\sim 60-80$ per cent of the sources classified as AGN, but only overlap for $\sim 44$ per cent of the total. Hence, together they account for almost all, 90 per cent (160/178), of the radio sources classed as AGN. The sensitivity of our $K$-band and 24- $\mu \mathrm{m}$ observations are generally deep enough that we are not biased by non-detections in these bands as that would put a source clearly out of the SFG regime, except possibly for the mid-IR/radio ratio where above redshift 1 we get a few $24 \mu \mathrm{m}$ non-detections in the SFG regime for the very faintest radio sources. The morphology and spectral index methods are not as powerful (see Table 1), being primarily limited by the sensitivity and coverage of the current observations. Of the sources classed as AGN, these discriminators are together responsible for classifying 35.9 per cent, far less than that due to the flux ratio cuts, but these two other selection criteria tend to agree with the flux ratio methods.

We also investigated the effect of systematically varying the two main discriminators, the flux density ratios plotted against redshift, by $\pm 1 \sigma(=0.12 \mathrm{dex})$. When both cuts were changed so as to classify more radio sources as SFGs we found a swing (i.e. change in AGN/SFG distribution) of 4.2 per cent in the number of SFGs from the parent sample and when changed so as to increase the number of AGN we found a swing of 5.6 per cent. These values are on par with the Poisson statistics expected from dividing the parent sample into two roughly equal populations ( $\sim 6$ per cent). It is possible that some or many of the SFGs may contain AGN, but that these AGN

Table 2. Results of the discrimination of sub-mJy radio sources. The raw numbers are presented for each galaxy type as well the number of with spectroscopic, photometric or unknown redshifts. We also present the number of different sources corrected for incompleteness in the radio survey and the percentage of the total number of sources.

\begin{tabular}{ccccc}
\hline Classification & $N$ & $N_{\text {spec }} / N_{\text {phot }} / N_{?}$ & $N_{\text {corr }}$ & Percentage \\
\hline AGN & 178 & $31 / 88 / 59$ & 244 & 35.8 \\
SFGs & 269 & $131 / 138 / 0$ & 436 & 64.0 \\
Stars & 1 & $1 / 0 / 0$ & 1 & 0.1 \\
\hline
\end{tabular}

Table 3. Break-down of the number of radio sources classified as AGN by each of our four discrimination methods. The columns and rows correspond to the four methods of AGN/SFG discrimination used in Sections 3.13.4: radio morphology, radio spectral index, mid-IR/radio flux density ratio and radio/near-IR flux density ratio. The diagonal represents the number of sources classified as AGN by just the corresponding discriminator. The lower left-hand side of the table, below the diagonal, gives the number of sources flagged as AGN for a particular combination of discriminators.

\begin{tabular}{ccccc}
\hline Classification & Morphology & $\alpha$ & Mid-IR & Near-IR \\
\hline Morphology & 44 & - & - & - \\
$\alpha$ & 12 & 32 & - & - \\
Mid-IR & 27 & 16 & 102 & - \\
Near-IR & 30 & 15 & 79 & 137 \\
\hline
\end{tabular}

do not contribute strongly to the radio emission, e.g. the 'IR-excess' sources in Fig. 1(b). It is also possible that some radio SFG are really radio AGN, especially as some of the discrimination methods do not work so well at faint flux densities. The possible number of AGN interlopers is difficult to quantify other than varying the discrimination criteria (see previous paragraph) without superior data.

\subsubsection{Radio sources with faint optical or near-IR detections}

Determining the nature of faint radio sources with weak or no detections at other wavelengths is naturally difficult. We have 60 sources from the parent sample where we are not able to determine a redshift, spectroscopic or photometric. In fact, 31 of these sources have no detections, $>3 \sigma$, at any other wavelength. The radio sources with unknown redshifts are included in Fig. 1 (both panels) at $z=0$, either as upper/lower limits or detections. Their positions in these plots suggest that they would be mainly classified as AGN whatever their redshift, especially in Fig. 1(a), where our AGN/SFG cut-off has only a weak dependence on redshift. We considered the possibility that they are low-mass SFGs at $z \sim 1$, i.e. not detected in our deep $K$ band of IRAC data. Such an object would have an SFR of $\sim 100 \mathrm{M}_{\odot} \mathrm{yr}^{-1}$, but a stellar mass $\lesssim 10^{8.5} \mathrm{M}_{\odot}$. A few sources with characteristics close to these values have been seen, e.g. the hosts of GRBs, but the SFR and non-detection in our $B$-band observations implies UV extinction of $A_{\mathrm{V}} \sim 8$ over the whole galaxy. While we may have missed a couple of extreme star-forming sources it is unlikely that many of these sources are starbursts at moderate redshift.

We present the radio flux density verses $I$-band magnitude distribution in Fig. 2. This plot shows that the brightest radio sources $\left[\log \left(S_{1.4 \mathrm{GHz}} \mu \mathrm{Jy}^{-1}\right)>2.5\right]$ are mainly AGN, but with a population of SFGs coming in at lower radio flux densities and brighter optical magnitudes than the AGN. This result also suggests that the optically unidentified and faint sources are most likely to be AGN (e.g. at high redshift and obscured). At $z \geqslant 1$ sources would appear compact enough that we should detect intrinsically bright, but low surface brightness galaxies at the depths of our data which have a typical seeing of 1 arcsec. Below $z=2.2$ we expect to detect all

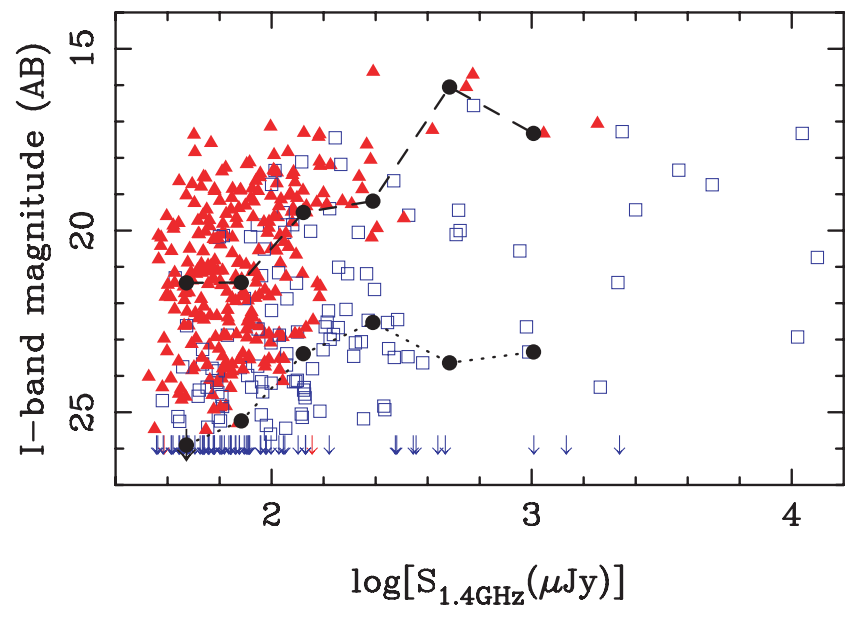

Figure 2. The 1.4-GHz radio flux density plotted against $I$-band magnitude of all our sources separated into AGN (blue squares) and SFGs (red triangles). Radio sources undetected in $I$ band above $3 \sigma$ are marked as upper limits at $I \sim 25.9$. The lines represent the median $I$-band magnitude for the SFGs (dashed line) and AGN (dotted line) as a function of flux density. 
sources with $\log \left(L_{2.2 \mu \mathrm{m}} / \mathrm{L}_{\odot}\right)>10$, in our near-IR or IRAC data, corresponding to quite low stellar masses [i.e. $\log \left(M / \mathrm{M}_{\odot}\right) \sim 10$ depending on the exact mass-to-light ratio]. We plot the median $I$ band magnitude of the SFGs and AGN in Fig. 2 which both decrease toward fainter radio flux densities. We believe the parent sample to be reliable as we would expect 0.03 of sources to be spurious in pure Gaussian noise, although the noise in radio maps is not completely Gaussian. In S04 we carefully examined visually all the sources to remove those that were clearly due to artefacts from the radio reduction. We cannot rule out the possibility that one or two sources at low S/N and with no optical remain spurious, but our results would not change significantly if this were the case.

Fig. 2 shows that the optical properties of the parent sample do change with radio flux density contrary to the results of Simpson et al. (2006) who do not see a decrease in the median magnitude with decreasing radio flux density. The difference between the results here and those of Simpson et al. (2006) can mainly be explained by the fact we have deeper radio data (by 0.5 dex) and the trend we see is strongest at the faintest flux densities. Results at the highest flux densities are likely affected by small number statistics. Hence, we conclude that most, if not all, of the radio sources with optically very faint counterparts are high-redshift radio galaxies (their optical faintness implying they are obscured, type II AGN). They are likely to be similar to the classical high-redshift radio galaxies, but less luminous (i.e. in the $24 \leqslant \log \left(L_{1.4 \mathrm{GHz}} / \mathrm{W} \mathrm{Hz}^{-1}\right) \leqslant 26$ range Sajina et al. 2007).

\section{RADIO SOURCE COUNTS BY TYPE}

The total Euclidean normalized radio source counts from the $13^{\mathrm{H}}$ field were presented in S04 where we found the counts to be in agreement with the many other published counts at similar flux densities. However, a scatter between the different counts from different surveys was found which was particularly strong around 200-300 $\mu \mathrm{Jy}$ with the counts from the HDF-N (Richards 2000) being significantly low. This scatter was attributed to sample variation due to large-scale structure. An independent reanalysis of the HDF-N counts by Biggs \& Ivison (2006) revised them upwards, but a smaller scatter due to sample variance nevertheless remains. However, we note that the counts from the $13^{\mathrm{H}}$ field, Phoenix Deep Field Survey (Hopkins et al. 2003), HDF-S (Huynh et al. 2005) and the three fields from Biggs \& Ivison (2006) are consistent with only a 10-20 per cent scatter over the 70-500 $\mu$ Jy flux density range.

We can now recalculate the source counts at $1.4 \mathrm{GHz}$ separately by type, AGN and SFG, correcting for incompleteness of the radio survey as in S04. As in the original presentation of these counts we only take sources above $5 \sigma$. Uncertainties include Poisson statistics and sample variance (which we discuss in Section 5.2).

The counts by type are presented in Table 4 and Fig. 3. We use slightly different binning than in S04 to obtain the most uniform distribution of numbers for both AGN and SFG populations per bin. We find that there is a rapid rise in the contribution to the counts from SFGs as expected from modelling of the counts (Rowan-Robinson et al. 1993; Hopkins et al. 1998; Hopkins 2004; S04; Huynh et al. 2005). The SFGs are the more numerous population below $120 \mu \mathrm{Jy}$ although the AGN still contribute approximately one quarter of the total counts even in the faintest bin. So while SFGs dominate, AGN do have a small contribution to the upturn, but maybe not as much as suggested by Simpson et al. (2006). The fraction of SFGs in the two lowest flux density bins, $72 \pm 22$ per cent, is consistent with the $\sim 70$ per cent of $<0.1 \mathrm{mJy}$ sources found to be SFGs by Muxlow et al. (2005) in high-resolution (0.2-0.5 arcsec) MERLIN + VLA
Table 4. Euclidean normalized 1.4-GHz source counts: total and by galaxy type. The first column shows the range of flux densities of each bin. The second column is the middle of the bin in log space. The last three columns indicate the Euclidean normalized source counts: total and for AGN and SFGs separately for the first time with their associated uncertainties.

\begin{tabular}{ccccc}
\hline $\begin{array}{c}\text { Flux range } \\
(\mu \mathrm{Jy})\end{array}$ & $\begin{array}{c}\text { Flux } \\
(\mu \mathrm{Jy})\end{array}$ & Total & $\begin{array}{c}\text { Counts } \\
\left(\mathrm{sr}^{-1} \mathrm{Jy}^{1.5}\right) \\
\mathrm{AGN}\end{array}$ & SFGs \\
\hline $37.5-55.7$ & 45.7 & $3.48 \pm 0.53$ & $0.98 \pm 0.22$ & $2.50 \pm 0.41$ \\
$55.7-89.6$ & 70.7 & $3.94 \pm 0.54$ & $1.11 \pm 0.21$ & $2.83 \pm 0.41$ \\
$89.6-156.1$ & 118.3 & $4.12 \pm 0.42$ & $1.61 \pm 0.24$ & $2.51 \pm 0.32$ \\
$156.1-294.1$ & 214.2 & $2.73 \pm 0.46$ & $1.82 \pm 0.37$ & $0.91 \pm 0.27$ \\
$294.1-600.0$ & 420.1 & $3.46 \pm 0.79$ & $2.53 \pm 0.64$ & $0.99 \pm 0.50$ \\
\hline
\end{tabular}

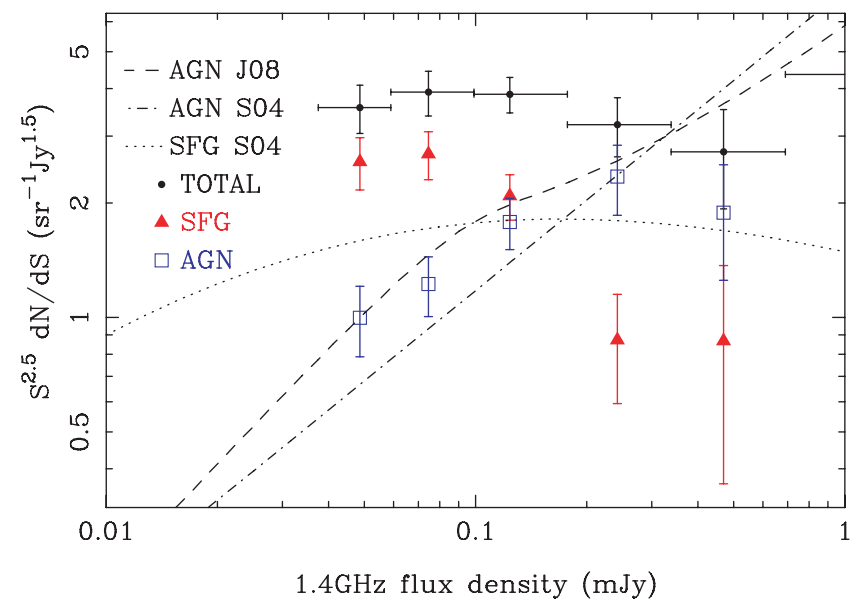

Figure 3. The Euclidean normalized 1.4-GHz source counts for the $13^{\mathrm{H}}$ deep survey: total counts (filed circles), SFGs (triangles) and AGN (open squares). Error bars represent uncertainties derived from Poisson statistics and sample variance. We overlay AGN and SFG models from S04 and Jarvis et al. (in preparation). Note that a large part of the upturn in counts above the extrapolation of earlier AGN models (S04 etc.) from brighter flux densities is explained by SFGs, but that the AGN population partly contributes (about one quarter) to the upturn even in the faintest bins.

imaging of the HDF-N by. We note that no sources were detected between 27 and $40 \mu \mathrm{Jy}(\mathrm{S} / \mathrm{N}>8 \sigma)$ in the inner $3.4 \times 3.8$ arcmin in that work. Those authors inferred that the $\sim 8$ sources expected from the extrapolation of the source counts were resolved out on scales $>0.5$ arcsec and hence, in our opinion, are likely to be mostly SFGs, i.e. a SFG fraction close to 100 per cent.

The sharp disconnect in the number of SFGs from 0.2 to $0.1 \mathrm{mJy}$ is perhaps due to the fact that our AGN selection method may be more efficient at higher flux densities, but it most likely represents the sharp rise in the luminosity of a typical SFG from $z=0$ to 1 and the small number statistics in the highest flux density bins. The effect of slightly varying the flux ratio cuts in the two main AGN/SFG discrimination methods (as described in Section 3.5) does not qualitatively change the results in Fig. 3. When changing the cut-offs in flux ratio by 0.12 dex in favour of increased SFG numbers the swing in the faintest flux density bin is 5.2 per cent and when changing them in favour of the AGN the swing in this bin 10.4 per cent. These swings are less than the quoted uncertainties on the source counts in this bin, $\sim 20$ per cent.

We overlay some models for the AGN and SFG contribution to the radio source counts. We show the SFG and AGN model from S04 
and the AGN model courtesy of M. Jarvis and R. Wilman, based on Jarvis \& Rawlings (2004). The S04 AGN model is basically an extrapolation from higher flux densities of the models used in Dunlop \& Peacock (1990) and Hopkins et al. (1998) and hence not necessarily valid for these low flux densities although they were the best available at the time. The Jarvis AGN model matches the observed AGN counts well and includes a component of radio-quiet QSOs based on the evolution of the X-ray AGN LF. This model appears to follow reasonably well the increase in AGN above the extrapolation of older models, like that used in S04, from higher flux densities. The S04 SFG model is derived from the local LF of SFGs (from Sadler et al. 2002) with luminosity evolution of the form $(1+z)^{2.5}$ to $z=2$ (and constant thereafter), and does not fit the source counts well by overpredicting the SFG counts at the bright end and underpredicting them at the faint end. The discrepancy at the bright end may be due to the small volume probed at low-redshift where the brightest SFGs are likely to lie.

\section{PROPERTIES OF THE RADIO STAR-FORMING GALAXY POPULATION}

\subsection{Star formation rates and stellar masses}

The conversion of radio luminosity to SFR (Bell 2003) relies on the well known radio-IR relation (e.g. Yun et al. 2001). While we show in Appendix A that the radio-total IR relation does indeed hold to high redshifts/high luminosities, to derive SFRs from the radio/IR correlation in such situations requires two subtle corrections. The first is that in determining the local correlation, Yun et al. (2001) did not apply $k$-corrections, which become significant for the highest luminosity galaxies in their sample. The second issue is that some of these galaxies have relatively flat radio spectra, so correcting those observed at high redshift back to $1.4 \mathrm{GHz}$ using a nominal slope of -0.7 does not provide a good estimate. When nominal $k$-corrections are applied to the Yun et al. sample, the best-fitting ULIRG ratio of $L_{60 \mathrm{um}} / L_{1.4 \mathrm{GHz}}$ is $\sim 140$. We have used the observations of Condon et al. (1991) to determine a more accurate slope. We find a final ratio of $L_{60 \mu \mathrm{m}} / L_{1.4 \mathrm{GHz}}=128$ for a galaxy at $z \sim 2$ observed at $1.4 \mathrm{GHz}$ and corrected as if it had a slope of -0.7 . This result would imply that the SFRs estimated above using the standard local ratio are underestimated by about 0.1 dex, a correction we have applied in our analysis. After these corrections and our choice of a Kroupa (2001) initial mass function (IMF), our conversion from radio luminosity to SFR is 0.07 dex less than that of Bell (2003), i.e. for a given radio luminosity we assume an SFR 0.84 times that predicted by Bell (2003).

We plot the SFRs of our SFG sample against redshift in Fig. 4(a) using different symbols for objects of different stellar masses. Stellar mass estimates are calculated for the $13^{\mathrm{H}}$ sample of SFGs by normalizing a M82 SED to our IRAC $3.6 \mu \mathrm{m}$ fluxes ( $K$ band for eight sources not covered by the Spitzer data) and using a Kroupa (2001) IMF to determine a rest-frame $H$-band luminosities and assuming a M82 mass-to-( $H$-band)-light ratio. Similarly, we derived stellar masses for the $6 \mathrm{df}-\mathrm{NVSS}$ sample from their observed $K$-band magnitudes assuming the same M82 SED and mass-to-light ratio. Clearly using one mass-to-light ratio for a sample of galaxies with a range of SFRs, amongst other properties, is not ideal, but this approach suffices for the current investigation. The possible selection effects of this choice are discussed in Section 5.3.1.

We find two striking results. First, there are many very high SFR, $>300 \mathrm{M}_{\odot} \mathrm{yr}^{-1}$, sources above $z=1$ compared to the local Universe. The 6dF-NVSS Survey covers a large area of the sky, 17 per cent equivalent to $\sim 7 \mathrm{Gpc}^{3}$ to $z=0.3$, hence is not biased against detecting rare, exceptionally high SFR sources. Therefore, the lack of exceptionally high SFR sources locally is not a volume selection effect and the presence of very high SFR galaxies at high redshift is observed in surveys at other wavelengths (e.g. in the submillimetre Chapman et al. 2005; Muxlow et al. 2005; Pope et al. 2006). Secondly, all our high SFR galaxies have very high stellar masses. We discuss this result more within the framework of current galaxy evolution models in Section 5.3.

We can compare the SFR against stellar mass for both our sample of SFGs and the 6df-NVSS sample in Fig. 4(b) and we find that a trend of SFR correlating with stellar mass is apparent in both samples (i.e. from the local universe up to $z \lesssim 3$ ). As radio flux limited surveys will always sample the highest SFR objects at any given redshift, this result is consistent with the idea that there is an upper limit to the SFR of a galaxy of a given mass. We see a similar trend in the results of Noeske et al. (2007) who find a 'main-sequence' relation between SFR and stellar mass at a given redshift with increasing
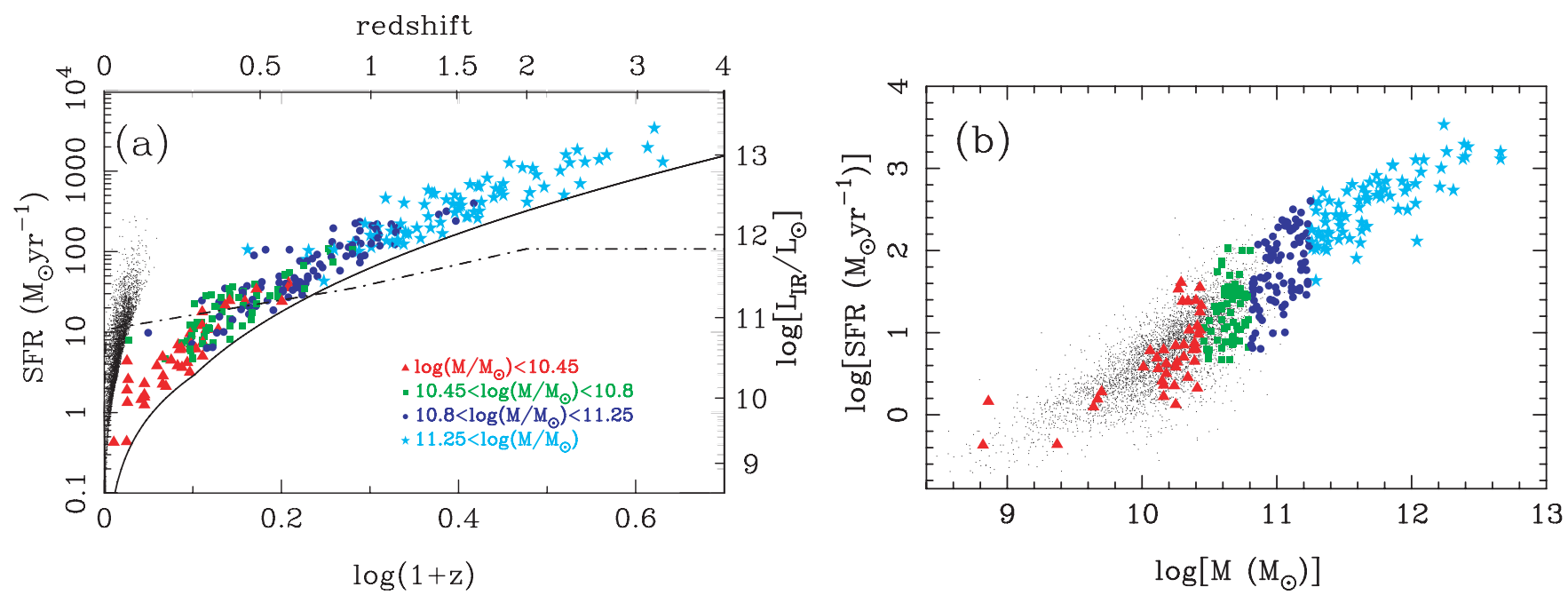

Figure 4. SFRs of the radio SFGs from the $13^{\mathrm{H}}$ field plotted against redshift (a) and stellar mass (b). The different symbols represent different stellar masses ranges as indicated in panel (a). In panel (a), the solid line represents the 1.4-GHz detection limit and the dot-dashed line indicates the 10 times $L \star$ of the radio star-forming LF taking a luminosity evolution of $Q=2.5$ which we use in Section 5.3. The black dots are the SFGs from the local 6dF-NVSS sample. 
SFR observed at higher stellar masses. The hypothesis that lower mass galaxies can only achieve lower maximum SFRs could be explained by the fact that more massive galaxies generally have more baryons in gas and dust which can act as raw material for the star formation. Potentially some of the very high radio luminosities could be overestimated due to uncertainties in both the photometric redshift and $k$-correction (i.e. radio spectral index). Our radio/nearIR flux ratio cut does affect the number of sources detected with high SFR to mass ratio, at high masses/SFR/redshift. However, we have demonstrated in Section 3.5 that by varying this cut by $1 \sigma=$ \pm 0.12 dex does not affect the results our AGN/SFG discrimination very much. We may have one or two AGN interlopers, but we are likely complete in a statistical sense.

\subsection{Star formation history of the Universe across $0<z<3$}

The SFGs are of great interest as their radio luminosity provides a relatively unbiased measure of their SFR independent of the effects of dust. Using this tracer of star formation we can probe the global cosmic SFR (e.g. Lilly et al. 1996; Madau et al. 1996). We restricted the sample to all sources with $z \leqslant 3$ as we only detect two SFGs above $z=3$.

The SFR densities are calculated using the standard $1 / V_{\max }$ method which allows for the change in detection limit across the relatively broad redshift bins. The redshift bins are chosen to be equal in size in $\log (1+z)$ space and to have at least 50 sources per bin apart from the highest redshift bin which has 13 sources. The redshift bins are deliberately left relatively wide to mitigate largescale structure/sample variance, Poisson statistics and any possible systematics in the uncertainties of the photometric redshifts. The total SFRD in each redshift bin requires two corrections:

(i) Radio survey incompleteness: The radio survey is not complete down to the $30 \mu \mathrm{Jy}$ detection limit at $1.4 \mathrm{GHz}$, mainly due to the attenuation of the primary beam of the VLA away from the pointing centre, but also due to other instrumental effects such as bandwidth smearing. Hence the radio sources at low $\mathrm{S} / \mathrm{N}, \lesssim 100 \mu \mathrm{Jy}$, need to be corrected by a weighting factor representing the sources not detected in our survey. The weighting factor for each source is a measure of the sky area sampled by the $1.4-\mathrm{GHz}$ survey at the flux density of the source. This correction factor is a function of flux density and position away from the pointing centre, i.e. our detection limit is $\sim 60 \mu \mathrm{Jy}$ at the edge of the field. This correction is described in full in S04 and we apply the individual weightings from that work to each source.

(ii) Star-forming radio sources below the nominal 1.4-GHz VLA detection limit: As shown in panel (a) of Fig. 4, our survey primarily probes only the high-luminosity end of the the SFG LF, especially at high redshift. Any estimate of the SFRD needs to include the contribution from sources further down the LF and below the detection limit. Clearly the LF must evolve rapidly given the fits to the source counts (Hopkins et al. 1998; S04) and the appearance of very high SFR sources at $z>1$.

The evolution of the SFG LF remains slightly uncertain, but most results find a luminosity evolution of $Q=2-3$, using the form ( $1+$ $z)^{Q}$, with negligible density evolution (Rowan-Robinson et al. 1993; Hopkins 2004; S04; Huynh et al. 2005; Moss et al. 2007). Haarsma et al. (2000) use a different functional form of the evolution with more free parameters, but find quantitatively the same behaviours for luminosity and density evolution as the authors above. However, Haarsma et al. (2000) do not provide uncertainties for their fitted parameters. As there is no consensus on the evolution parameter in
Table 5. SFRD at different epochs. The first column represents the size of the redshift bin. The second column indicates the middle of the bin in $\log (1+z)$. The third column gives the number of SFGs in each redshift bin. The fourth column indicates the multiplicative correction due to the part of the LF below the detection limit using the SFG LF with luminosity evolution of the form $(1+z)^{2.5 \pm 0.5}$. The fifth column presents the comoving SFRD derived from the SFGs and the uncertainty.

\begin{tabular}{ccccc}
\hline $\begin{array}{c}\text { Redshift } \\
\text { range }\end{array}$ & $\langle z\rangle$ & $\#$ & LF correction (per cent) & $\begin{array}{c}\mathrm{SFRD} \\
\log \left(\mathrm{M}_{\odot} \mathrm{yr}^{-1} \mathrm{Mpc}^{-3}\right)\end{array}$ \\
\hline $0.10-0.52$ & 0.29 & 104 & $1.35_{-3.8}^{+3.5}$ & $-1.48_{-0.11}^{+0.09}$ \\
$0.52-1.10$ & 0.79 & 87 & $2.68_{-20.0}^{+21.0}$ & $-1.07_{-0.16}^{+0.12}$ \\
$1.10-1.90$ & 1.47 & 54 & $4.60_{-38.1}^{+48.3}$ & $-0.83_{-0.26}^{+0.19}$ \\
$1.90-3.00$ & 2.40 & 15 & $6.43_{-53.2}^{+81.6}$ & $-0.92_{-0.43}^{+0.27}$ \\
\hline
\end{tabular}

the literature, or the exact form of the evolution, we take a representative value of $Q=2.5 \pm 0.5$, which is consistent with preliminary results from fitting the radio LF (D04), and $P=0$ for the luminosity and density evolution parameters, respectively. We then use the local radio SFG LF of Mauch \& Sadler (2007). We use these evolution parameters to calculate the fraction of the luminosity density below our nominal detection limit for each redshift bin. We give the corresponding multiplicative correction factors and associated uncertainties in Table 5.

The uncertainties include the following effects.

(i) Poisson uncertainties: We chose the redshift bin size to include at least $\sim 50$ sources per bin apart from the highest redshift bin.

(ii) Uncertainty in the evolution of the LF: As mentioned earlier in this section, the amount of luminosity evolution of the LF remains uncertain so we have used a representative value of $Q=2.5 \pm 0.5$. Hence we include this uncertainty when derived the uncertainty of the SFRD. The effect of including this factor gets stronger at higher redshifts as can be seen in Fig. 5 and the LF correction factor in Table 5 .

(iii) Sample variance: Sometimes referred to as cosmic variance, this effect is really quantifying how representative our narrow field is of the whole sky. The source counts between different fields do vary by $10-20$ per cent as discussed earlier, but accurately measuring the field to field variation between different surveys is tricky due to different methods used and in some cases different telescopes to correct for instrumental effects that dominate at the faint end. This issue needs to be addressed by the different teams working on deep radio surveys although we previously noted in Section 4 that several major surveys are now in reasonable agreement. Hence, to allow for sample variance we simply add a further 20 per cent uncertainty.

We further investigate the effects of changing the two main discriminators (the two flux density ratios as described in Section 3.5). When changing both these cuts in favour of the SFGs we get an increase in the densities, but this is barely noticeable in the lowest redshift bin and increases the highest redshift bin by just 10 per cent When changing the cuts the other way, in favour of the AGN, the densities decrease, but only by a few per cent at the lowest redshift and by 23 per cent in the highest redshift bin. Clearly the effect of changing the selection criteria is strongest in the highest redshift bin, but this result is not unexpected since this bin typically contains the lowest $\mathrm{S} / \mathrm{N}$ radio sources and lowest number of sources. The flux ratio discriminators are least good at the highest redshifts, and the radio morphology/spectral index methods cannot be applied 


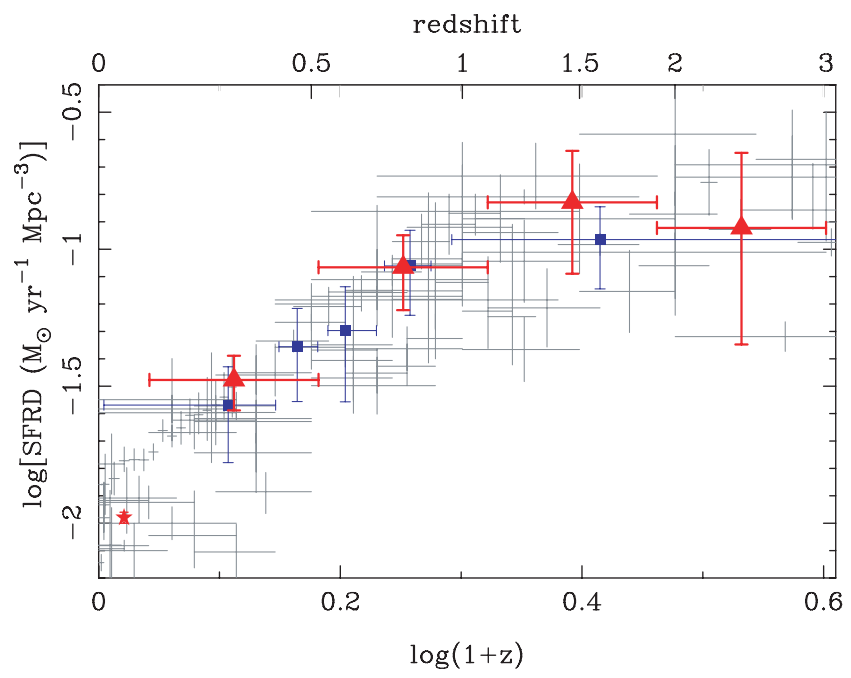

Figure 5. The comoving SFRD of the Universe as a function of redshift from this work (red triangles) and other surveys. The horizontal error bars represent the width of the bins and vertical error bars represent the combined uncertainties (see text for details). The SFRD from a selection of other methods (UV, $\mathrm{H} \alpha$, far-IR, etc.) taken from a compilation in Hopkins (2004) are marked by grey lines indicating bin widths and associated uncertainties. From this sample we have highlighted (as blue squares) the results of Haarsma et al. (2000) which are also derived from radio observations. The local $(z=0.05)$ value of the radio derived SFRD from (Mauch \& Sadler 2007) is plotted as a red star with uncertainties smaller than the symbol size.

because most objects are too faint. Furthermore, these changes are generally smaller than the combined uncertainties from the effects listed above and hence indicate that the AGN/SFG discrimination criteria used in Section 3 are not the largest uncertainty in deriving the SFRD at the highest redshift.

The results of our determination of the comoving SFRD of the Universe as a function of redshift are presented in Table 5 and Fig. 5. These results agree well with the multiwavelength sample from Hopkins (2004), converted to a Kroupa IMF, showing the rapid rise from the local value to a value over an order of magnitude larger at $z \geqslant 1$, followed by a flattening above $z \sim 1.5$. We highlight the results of Haarsma et al. (2000), as blue squares corrected to the same IMF and cosmology, who also derived SFR comoving density from a deep VLA observation of several fields. Their results are consistent with ours at all redshifts. Haarsma et al. (2000) perform similar corrections for instrumental effects and for sources below the nominal detection limit, but have very different AGN/SFG discrimination methods and less sophisticated methods of redshift determination (i.e. for sources without spectroscopic redshifts those authors use estimates from $I$ or $H K^{\prime}$ magnitudes, or have random assignments). Their uncertainties are relatively small as they do not include uncertainties in sample variance. Our results are also consistent with the results of Ivison et al. (2007b) who use stacking of different galaxy types in the radio to derive SFR densities at different redshifts. The largest uncertainty in our work is that from the evolution of the LF, necessary to correct for the unsampled part of the LF.

\subsection{Contribution to star formation rate density by galaxy stellar mass}

Recent studies of the star formation history of the Universe have not only measured the rapid change in global SFRD, but also the demographics of the SFGs. Many authors have observed the evolution of the distribution of star formation moving high-mass galaxies at high redshift to lower mass galaxies at lower redshifts (e.g. Cowie et al. 1996; Panter, Heavens \& Jimenez 2004; Juneau et al. 2005; Noeske et al. 2007; Panter et al. 2007). This effect has been termed 'downsizing' and is analogous to (and possibly related to) the apparent shift of the peak number density of AGN to lower redshifts for lower X-ray luminosities (e.g. Ueda et al. 2003).

We are able to examine 'downsizing' too with our unique radio-selected sample of SFGs across $0<z<2$. In this section we chose to look just at the contribution from the $L_{1.4 \mathrm{GHz}}>$ $10 L_{\star}$ galaxies where we are mostly complete up to $z=2$, Fig. 4(a). The $L_{1.4 \mathrm{GHz}}>10 L_{\star}$ top end of the LF represents the top 28 per cent of the total SFRD at any redshift, for the best-fitting Mauch $\&$ Sadler (2007) LF assuming pure luminosity evolution. Hence we can divide each SFRD bin into the contribution by stellar mass including that of the local sample from Mauch \& Sadler (2007). We only show the fractional contribution by luminosity/SFRD in Fig. 6, but note that the distribution by number density is very similar. Hence, number density and luminosity density are both good tracers of downsizing.

While the sample shown in Fig. 6 represents about one quarter of the star formation occurring at a given cosmic epoch, the $L_{1.4 \mathrm{GHz}}>$ $10 L_{\star}$ sources are the most active at any redshift. We find that there is a dramatic change in the stellar mass of the galaxies contributing to this part of the LF. The highest redshift bin is dominated by massive galaxies, $\log \left(M / \mathrm{M}_{\odot}\right)>11.25$, although at this redshift we are not quite complete to $L_{1.4 \mathrm{GHz}}=10 L_{\star}$ and hence we mark points in this bin as limits. In the lowest redshift bin (from the $6 \mathrm{df}$ sample) we can see the dramatic, rapid rise in the number of the least massive galaxies. We are even able to see the rise and fall of the contribution from intermediate stellar mass ranges at successive intermediate redshift ranges.

These results are qualitatively similar to other recent results (e.g. Juneau et al. 2005; Panter et al. 2007), but are difficult to compare quantitatively as they differ in the selection details. The differences are largely due to the very different survey volumes probed

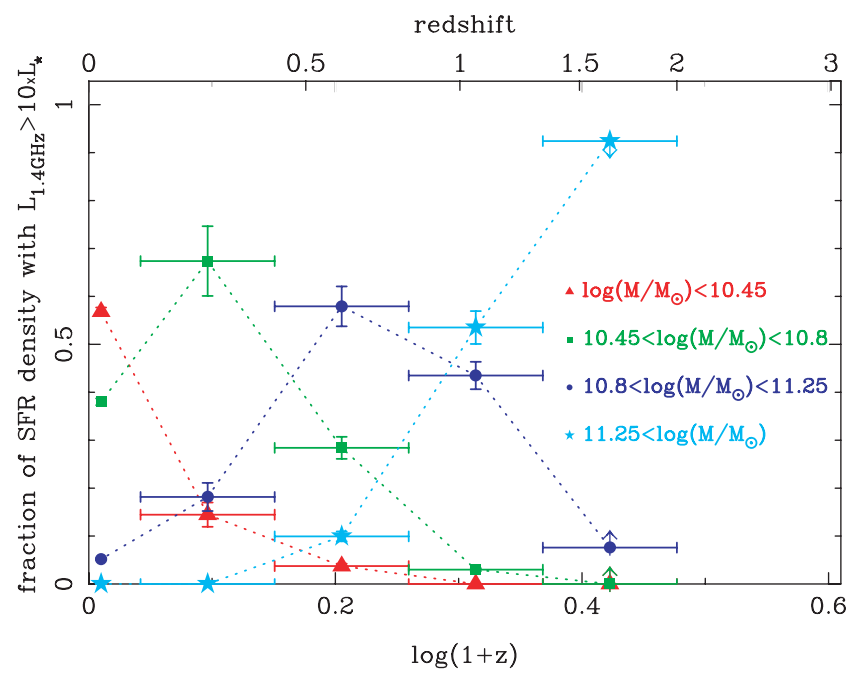

Figure 6. The contribution by luminosity/SFRD to the $L_{1.4 \mathrm{GHz}}>10 L_{\star}$ range of the SFG LF. SFGs with different mass ranges are indicated on the plot following our earlier convention. This high end of the LF (in the form from Saunders et al. 1990) represents about 28 per cent of the SFRD at any particular redshift. The points at $\log (1+z)=0.02$ are from the $6 \mathrm{dF}-\mathrm{NVSS}$ SFG sample and the other points at higher redshift are from the $13^{\mathrm{H}} \mathrm{SFG}$ sample. 
and methods of determining the star formation history. Juneau et al. (2005) reach lower SFRs determined from optical emission lines from sources in Gemini Deep Deep Survey (GDDS) over an area 20 times smaller than the work presented here, hence their sample includes far fewer of the rarer, extremely high SFR objects that we find. Panter et al. (2007) use the data from the Sloan Digital Sky Survey Third Data Release (covering a far greater area than our survey, $\sim 5200 \mathrm{deg}^{2}$ ) and model the galaxy spectra to determine the individual and global star formation histories.

What Fig. 6 truly shows us is the fall of the contribution by massive galaxies to the global SFRD. We cannot rule out that the contribution of the least massive to the total SFRD remains approximately constant over this redshift range. As the most massive galaxies are capable of the highest SFRs they dominate the upper end of the LF at the high redshift which we explore. It is likely that as these massive galaxies are capable of such high SFRs they use up their material to form stars very quickly and hence quash their SFR.

\subsubsection{Selection effects}

We are selecting our SFGs on an SFR indicator that is completely unbiased with respect to obscuration and with little selection on redshift beyond that implied by our radio flux density limit. At any redshift we are naturally detect just the most luminous, and hence highest SFR galaxies. One thing to consider is whether the apparent observed downsizing is simply an effect of missing lowmass galaxies in our high-redshift bins. SFGs in our lowest mass bin only become undetectable at $z \geqslant 3$ in our $K$ band or IRAC data. Low-mass SFGs at these redshifts with SFRs high enough to be included in our sample would extremely high specific SFRs (SSFRs) $\left(>10^{-8} \mathrm{yr}^{-1}\right)$ and we argue in Section 3.6.1 that we have are unlikely to have missed any such extreme sources. Hence, the low detection rate of low-mass galaxies in the high and intermediate redshift bins is not due to our detection limits.

One might argue that the trend of fewer high stellar mass galaxies at lower redshift may be a volume selection effect. However, we can compare the number of SFGs selected from their high SFR to that expected purely on mass selection. In the lowest redshift bin for the $13^{\mathrm{H}}$ sample, $0.1 \leqslant z \leqslant 0.4$, we would expect to detect $\sim 0.2$ galaxies in the highest mass bin in this volume simply using the fitted Schechter function to the local stellar mass function of SFGs from Panter et al. (2007) (assuming no evolution from $z$ $=0$, Fontana et al. 2006). This prediction is consistent with our result of no galaxies found in this bin. In the highest redshift bin we would expect to detect $\sim 40$ high-mass galaxies using the same method as before, i.e. assuming no evolution of the mass function. However, given that Fontana et al. (2006) suggest that this part of the mass function is not in place until $z \leqslant 1$ this estimate of $\sim 40$ may be an upper limit and hence is consistent with our result where we detect 31 high-mass galaxies in this redshift bin. These rough estimates have to be treated as such as the whole of the highest mass bin is above the knee of the stellar mass function where the space density changes most rapidly and is hardest to measure accurately. However, it is encouraging to see that number of massive galaxies varies approximately as expected. The ratio of massive to low-mass galaxies must, of course, decrease toward higher redshifts if there is hierarchical evolution in the mass function, but our selection on SFR shows the opposite trend with a higher ratio of high- to low-mass galaxies at higher redshift.

A further factor to consider is the choice of a single mass-to-light ratio for the entire SFG sample. Mass-to-light ratios can vary by over an order of magnitude from active to quiescent galaxies, but most of our sample are clearly quite active by any standard, hence any variation in the ratio will be much less than this amount. Photometric stellar masses used here are also quite model dependent unlike direct dynamical measurements. There is quite a debate concerning photometric stellar masses in the literature at the moment (e.g. Maraston 2005). We note, though, that relative photometric stellar masses are more accurate than absolute photometric stellar masses so the general trends examined in this section and the next are genuine cosmological effects.

\subsection{Characteristic times}

The SSFR of a galaxy is defined as its star formation rate per unit stellar mass, and is representative of how active a galaxy is compared to its past properties. For all but the most massive galaxies our survey is biased against low SSFRs as we are selecting on SFR only. The inverse of the SSFR has been referred to as the characteristic timescale (e.g. Juneau et al. 2005). This number has units of time and gives an idea of the nature of the star formation going on in the galaxy. For example if at a given redshift the characteristic time of a galaxy is less than the age of the Universe (or the time since the galaxy formed) then the current SFR is higher than the historic mean SFR and the galaxy can be said to be in a 'burst mode'. Conversely if the characteristic time is greater than the age of the Universe at a given time then the observed SFR is lower than the mean historic SFR and the galaxy can be said to be in a 'quiescent' mode. In Fig. 7 we plot the characteristic time as a function of redshift and along with the time since the big bang, $z=5$ and 3. Most of our SFGs can be classified as being in 'burst mode', although this classification becomes less certain at high redshift as it more strongly depends on the redshift of galaxy formation. This result implies that the current observed SFRs in most of the galaxies selected here are likely due to some trigger event beyond the initial gravitational collapse and

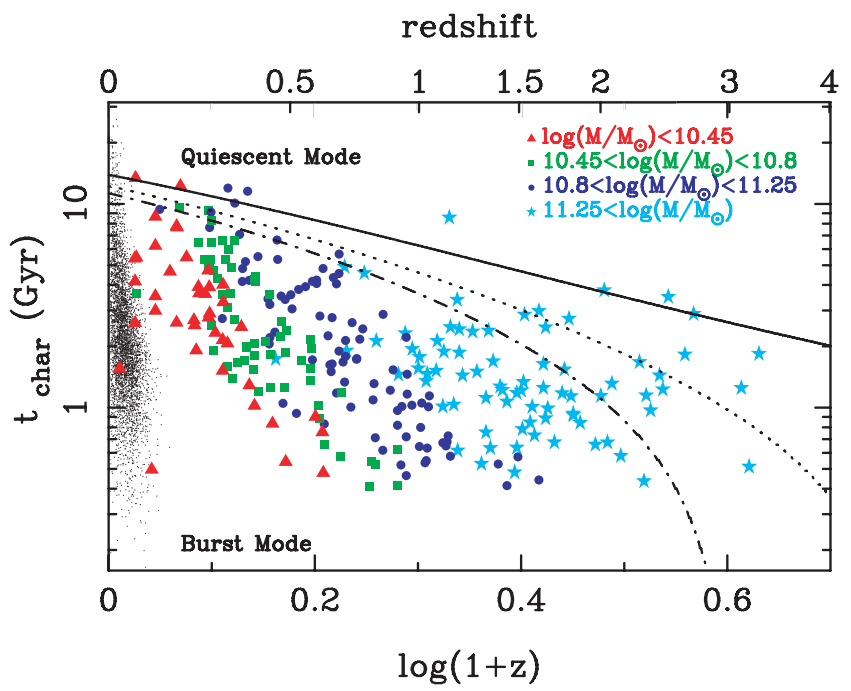

Figure 7. The 'characteristic time' (the inverse of the SSFR) of the radio SFGs plotted against redshift. The slowly decreasing solid line indicates the age of the Universe at a given redshift and the dotted (dot-dashed) line indicates the time since $z=5(3)$. Galaxies below these lines are forming stars at a faster rate than they were in the past and are said to be in a 'burst mode' and those galaxies above the line are said to be in 'quiescent mode'. For reference, the Milky Way has a characteristic time-scale of $\sim 100 \mathrm{Gyr}$ putting it firmly in the quiescent region of the figure (and off the plot), whilst M82 has a characteristic time of $\sim 0.7 \mathrm{Gyr}$, well in the local burst region. 
formation of the galaxy, perhaps AGN activity or galaxy-galaxy interaction. The fact that we detect the most SFGs in 'burst mode' is simply a function of the depth of the survey of the parent sample. All but the lowest redshift sources have SFR $>10 \mathrm{M}_{\odot} \mathrm{yr}^{-1}$, already an elevated value for most local galaxies. A much lower radio flux density limit is necessary to detect significant numbers of 'quiescent' galaxies at any kind of distance.

\section{CONCLUSIONS}

We present the analysis of a multiwavelength follow-up to one of the deepest radio surveys currently published. These data provide spectroscopic and photometric redshifts which are vital in discerning the nature of the sub-mJy radio population. With a philosophy of minimizing the assumptions about the nature of this population we consider four discriminators between AGN and SFGs based on purely the radio, or relative radio, properties of each source. These four discriminators are: radio morphology, radio spectral index, radio/near-IR and mid-IR/radio flux density ratios. We find that 178 objects in the parent sample were classified as AGN by at least one of our AGN discriminators and $\sim 90$ per cent of these are classified by at least one of the flux ratio methods. Our radio-selected parent sample contains a roughly 40/60 mix of AGN and SFGs, but with AGN dominating at high radio flux densities and SFGs dominating at faint flux densities. By recalculating the Euclidean normalized source counts by source type we find that the upturn of the counts below $1 \mathrm{mJy}$ is mainly due to SFGs, but still with a $\sim 25$ per cent contribution from AGN at the faintest flux densities. This result is consistent with previous model fits to the source counts and estimates from ultradeep MERLIN imaging.

We find many galaxies at high redshift which have SFRs exceeding those found in the local universe and that these galaxies tended to be more massive than SFGs at lower redshift. We also find a trend of SFR correlating with stellar mass at all redshifts from the 6df-NVSS sample and the $13^{\mathrm{H}}$ sample, i.e. for a galaxy at a given redshift and stellar mass there appears to be an upper limit to the SFR possible. After correcting for radio SFGs below our nominal detection limit $(4 \sigma=30 \mu \mathrm{Jy}$ at $1.4 \mathrm{GHz})$, we derive the comoving SFRD as a function of redshift. We find our results are consistent with those derived by methods at other wavelengths. We are able to look at the population of sources in the top end of the luminosity $\left(L_{1.4 \mathrm{GHz}}>10 L_{\star}\right)$ as a function of redshift and find that the typical mass of the sources making up the top one quarter of the star-forming LF changes dramatically with redshift. We also find that the characteristic time of the SFGs was generally low implying an enhanced current SFR for most galaxies compared to their past mean SFR.

The radio morphology and radio spectral index discriminator methods should be just as powerful as the flux ratio methods if deeper high-resolution and multiwavelength data were available. The mid-IR/radio flux density ratio would be improved by deeper mid-IR data. Radio luminosity, not used as a discriminator in this work, will become a powerful discriminator when larger volumes are probed in deeper and wider surveys. Unless the radio sources undetected at any other wavelength are extremely obscured (i.e. $A_{\mathrm{V}} \geqslant$ 8), then we are able to reject the hypothesis that they are low-mass SFGs at $z \sim 1$ because none of these radio sources have detections in our sensitive $u, B$ and $g^{\prime}$-band imaging. Such sources are most likely high redshift $(z>2)$, obscured type II AGN.

These results imply that deeper future radio surveys from eVLA, eMERLIN, LOFAR and (eventually) SKA will mainly detect SFGs, not only high SFR objects at extreme redshifts, but also low- luminosity, more quiescent SFGs at the redshifts probed in this work, giving a better view of the star formation in the distant Universe from radio data. The fraction of AGN is still non-negligible hence discrimination between AGN and SFGs will be the principal challenge in exploiting future surveys. This discrimination is by no means easy and, at least at present, largely statistical. Future surveys will need to be backed up by multiwavelength data, particularly mid-IR and near-IR, photometry, to confidently separate the SFGs and AGN, as well as deeper low frequency and high-resolution radio data.

\section{ACKNOWLEDGMENTS}

We thank the referee for help improving the presentation of this paper. We thank V. Smolčić, P. Capak, K. Sheth, M. Huynh and R. Norris for useful discussions. We thank T. Mauch for help with the 6dF-NVSS data set. We thank M. Jarvis for providing his model prior to publication. We thank K. Gunn for much help in this project over the years. AH acknowledges support provided by the Australian Research Council in the form of a QEII Fellowship (DP0557850). This work is based in part on data obtained with the Spitzer Space Telescope, which is operated by the Jet Propulsion Laboratory, California Institute of Technology under a contract with NASA. Support for this work was provided by an award issued by JPL/Caltech. Partial support for this work was provided by contract 1255094 issued by JPL/Caltech to the University of Arizona.

MERLIN is a National Facility operated by the University of Manchester at Jodrell Bank Observatory on behalf of STFC. This research used the facilities of the Canadian Astronomy Data Centre operated by the National Research Council of Canada with the support of the Canadian Space Agency. This work is based in part on data products produced at the TERAPIX data center located at the Institut d'Astrophysique de Paris.

Based on observations obtained at the Canada-France-Hawaii Telescope (CFHT), which is operated by the National Research Council of Canada, the Institut National des Sciences de I'Univers of the Centre National de la Recherche Scientifique of France, and the University of Hawaii. Based on observations obtained with MegaPrime/MegaCam, a joint project of CFHT and CEA/DAPNIA, at the Canada-France-Hawaii Telescope (CFHT) which is operated by the National Research Council (NRC) of Canada, the Institute National des Sciences de I'Univers of the Centre National de la Recherche Scientifique of France, and the University of Hawaii. Based in part on data collected at Subaru Telescope, which is operated by the National Astronomical Observatory of Japan. The WHT and INT are operated on the island of La Palma by the Isaac Newton Group in the Spanish Observatorio del Roque de los Muchachos of the Institute de Astrofísica de Canarias. The United Kingdom Infrared Telescope is operated by the Joint Astronomy Centre on behalf of the Science and Technology Facilities Council of the U.K. Based on observations obtained at the Hale Telescope, Palomar Observatory, as part of a collaborative agreement between the California Institute of Technology, its divisions Caltech Optical Observatories and the Jet Propulsion Laboratory (operated for NASA), and Cornell University. Some of the data presented herein were obtained at the W.M. Keck Observatory, which is operated as a scientific partnership among the California Institute of Technology, the University of California and the National Aeronautics and Space Administration. The Observatory was made possible by the generous financial support of the W.M. Keck Foundation. These observations have been funded in part by the Optical Infrared Coordination network (OPTICON), a major international collaboration supported by the Research Infrastructures Programme of the 
European Commission's Sixth Framework Programme. The authors wish to recognize and acknowledge the very significant cultural role and reverence that the summit of Mauna Kea has always had within the indigenous Hawaiian community. We are most fortunate to have the opportunity to conduct observations from this mountain.

\section{REFERENCES}

Appleton P. N. et al., 2004, ApJS, 154, 147

Armus L. et al., 2007, ApJ, 656, 148

Barger A. J., Cowie L. L., Wang W.-H., 2007, ApJ, 654, 764

Bauer F. E., Alexander D. M., Brandt W. N., Schneider D. P., Treister E., Hornschemeier A. E., Garmire G. P., 2004, AJ, 128, 2048

Bell E. F., 2003, ApJ, 586, 794

Bertin E., Arnouts S., 1996, A\&AS, 117, 393

Beswick R. J., Muxlow T. W. B., Thrall H., Richards A. M. S., Garrington S. T., 2008, MNRAS, in press

Biggs A., Ivison R., 2006, MNRAS, 371, 963

Bolzonella M., Miralles J.-M., Pelló R., 2000, A\&A, 363, 476

Boyle B. J., Cornwell T. J., Middelberg E., Norris R. P., Appleton P. N., Smail I., 2007, MNRAS, 376, 1182

Chapman S. C., Blain A. W., Smail I., Ivison R. J., 2005, ApJ, 622, 772

Condon J. J., 1992, ARA\&A, 30, 575

Condon J. J., Huang Z.-P., Yin Q. F., Thuan T. X., 1991, ApJ, 378, 65

Cowie L. L., Songaila A., Hu E. M., Cohen J. G., 1996, AJ, 112, 839

Donley J. L., Rieke G. H., Rigby J. R., Pérez-González P. G., 2005, ApJ, 634,169

Dunlop J. S., Peacock J. A., 1990, MNRAS, 247, 19

Elvis M. et al., 1994, ApJS, 95, 1

Fanaroff B. L., Riley J. M., 1974, MNRAS, 167, 31

Farrah D., Afonso J., Efstathiou A., Rowan-Robinson M., Fox M., Clements D., 2003, MNRAS, 343, 585

Fazio G. G. et al., 2004, ApJS, 154, 10

Fomalont E. B., Kellermann K. I., Cowie L. L., Capak P., Barger A. J., Partridge R. B., Windhorst R. A., Richards E. A., 2006, ApJS, 167, 103

Fontana A. et al., 2006, A\&A, 459, 745

Garrett M. A., 2002, A\&A, 384, L19

Gawiser E. et al., 2006, ApJS, 162, 1

Haarsma D. B., Partridge R. B., Windhorst R. A., Richards E. A., 2000, ApJ, 544,641

Hopkins A. M., 2004, ApJ, 615, 209

Hopkins A. M., Mobasher B., Cram L., Rowan-Robinson M., 1998, MNRAS, 296, 839

Hopkins A. M., Afonso J., Chan B., Cram L. E., Georgakakis A., Mobasher B., 2003, AJ, 125, 465

Huynh M. T., Jackson C. A., Norris R. P., Prandoni I., 2005, AJ, 130, 1373

Huynh M. T., Jackson C. A., Norris R. P., 2007, AJ, 133, 1331

Ivison R. J. et al., 2007a, ApJ, 660, L77

Ivison R. J. et al., 2007b, MNRAS, 380, 199

Jarvis M. J., Rawlings S., 2004, New Astron. Rev., 48, 1173

Juneau S. et al., 2005, ApJ, 619, L135

Kellerman K. I., Sramek R., Schmidt M., Shaffer D. B., Green R., 1989, AJ, 98, 1195

Klamer I. J., Ekers R. D., Bryant J. J., Hunstead R. W., Sadler E. M., De Breuck C., 2006, MNRAS, 371, 852

Kovács A., Chapman S. C., Dowell C. D., Blain A. W., Ivison R. J., Smail I., Phillips T. G., 2006, ApJ, 650, 592

Kroupa P., 2001, MNRAS, 322, 231

Lacy M. et al., 2005, ApJS, 161, 41

Lacy M., Petric A. O., Sajina A., Canalizo G., Storrie-Lombardi L. J., Armus L., Fadda D., Marleau F. R., 2007, AJ, 133, 186

Lilly S. J., Fevre O. L., Hammer F., Crampton D., 1996, ApJ, 460, L1

McHardy I. M. et al., 1998, MNRAS, 295, 641

Madau P., Ferguson H. C., Dickinson M. E., Giavalisco M., Steidel C. C., Fruchter A., 1996, MNRAS, 283, 1388

Maraston C., 2005, MNRAS, 362, 799
Mauch T., Sadler E. M., 2007, MNRAS, 375, 931

Miller L., Peacock J. A., Mead A. R. G., 1990, MNRAS, 244, 207

Moss D., Seymour N., McHardy I. M., Dwelly T., Page M. J., Loaring N. S., 2007, MNRAS, 378, 995

Muxlow T. W. B. et al., 2005, MNRAS, 358, 1159

Noeske K. G. et al., 2007, ApJ, 660, L43

Panter B., Heavens A. F., Jimenez R., 2004, MNRAS, 355, 764

Panter B., Jimenez R., Heavens A. F., Charlot S., 2007, MNRAS, 378, 1550

Papovich C. et al., 2007, ApJ, 668, 45

Pope A. et al., 2006, MNRAS, 370, 1185

Richards E. A., 2000, ApJ, 533, 611

Rieke G. H. et al., 2004, ApJS, 154, 25

Rowan-Robinson M., Benn C. R., Lawrence A., McMahon R. G., Broadhurst T. J., 1993, MNRAS, 263, 123

Sadler E. M. et al., 2002, MNRAS, 329, 227

Saunders W., Rowan-Robinson M., Lawrence A., Efstathiou G., Kaiser N., Ellis R. S., Frenk C. S., 1990, MNRAS, 242, 318

Sajina A., Yan L., Lacy M., Huynh M. 2007, ApJ, 667, L17

Schinnerer E. et al., 2007, ApJS, 172, 46

Seymour N., 2002, PhD thesis, Univ. Southampton

Seymour N., McHardy I. M., Gunn K. F., 2004, MNRAS, 352, 131 (S04)

Simpson C. et al., 2006, MNRAS, 372, 741

Spergel D. et al., 2003, ApJS, 148, 175

Stern D., Spinrad H., 1999, PASP, 111, 1475

Stern D. et al., 2005, ApJ, 631, 163

Thompson T. A., Quataert E., Waxman E., Murray N., Martin C. L., 2006, ApJ, 645, 186

Ueda Y., Akiyama M., Ohta K., Miyaji T., 2003, ApJ, 598, 886

Vlahakis C., Eales S., Dunne L., 2007, MNRAS, 605, 1042

Werner M. W. et al., 2004, ApJS, 154, 1

Yun M. S., Carilli C. L., 2002, ApJ, 568, 88

Yun M. S., Reddy N. A., Condon J. J., 2001, ApJ, 554, 803

\section{APPENDIX A: REDSHIFT DEPENDENCE OF THE RADIO-IR RELATION}

The radio-IR relation refers to the very close correlation, over five orders of magnitude, of the radio and IR luminosities of SFGs (Condon et al. 1991; Yun \& Carilli 2002) as the radio and IR luminosities are directly related to star formation. To use radio data as a measure of star formation, we need to determine if the radio-IR relation, from which radio/SFR calibrations are ultimately derived, holds or changes above $z=1$ (it has been shown to hold up to $z \sim 1$ in the mid-IR; Appleton et al. 2004). In fact, two recent studies have indicated that a change is possible (Kovács et al. 2006; Vlahakis, Eales \& Dunne 2007). We proceeded somewhat differently from these studies to avoid two possible sources of bias. Firstly, we take a sample of radio-selected, high-redshift galaxies (which have ULIRG or near-ULIRG luminosity) and a sample of local ULIRGs only, since the relation has not shown to be luminosity independent into the ULIRG range (i.e. local samples from Yun et al. 2001 and Bell 2003 are restricted to sub-ULIRG sources, $\log \left(L_{1.4 \mathrm{GHz}} / \mathrm{W} \mathrm{Hz}^{-1}\right)$ $\leqslant 24)$. Secondly, we compared the radio and IR rest-frame wavelength fluxes between the local ULIRGs and high-redshift galaxies, since free-free absorption can flatten the radio spectrum and make extrapolations back to rest-frame $1.4 \mathrm{GHz}$ uncertain.

First, we assembled a local comparison sample of ULIRGs dominated by star formation (according to the studies of Farrah et al. 2003; Armus et al. 2007) and with radio measurements at least at 1.4 and $8.4 \mathrm{GHz}$ (Condon et al. 1991). There are seven galaxies that meet these requirements; Arp 220, IRAS 1056, IRAS 1211, IRAS 1434, IRAS 1525, IRAS 17208 and IRAS 2249. Fig. A1 shows their SEDs, normalized at $260 \mu \mathrm{m}$. We overplotted the measurements of Kovács et al. (2006) of high-redshift galaxies at $1.4 \mathrm{GHz}$ and 


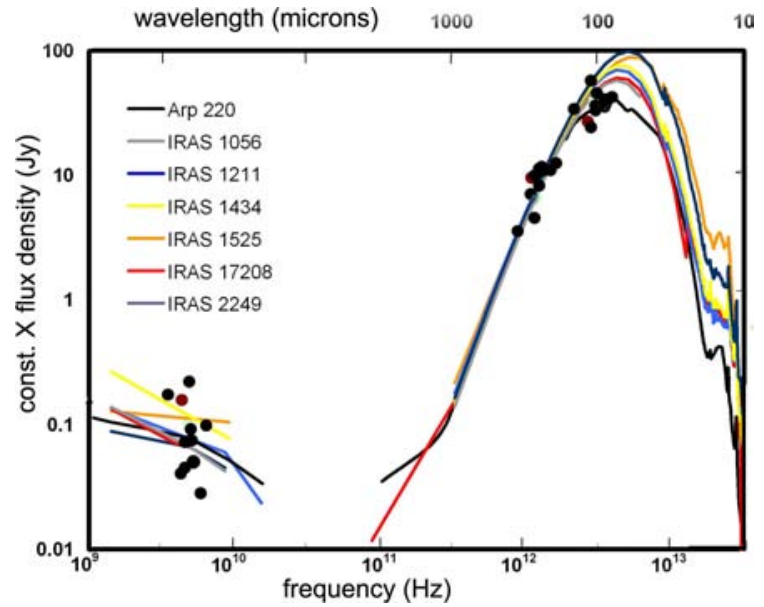

Figure A1. Observed rest-frame SEDs of local star-forming ULIRGs (as indicated in figure and normalized at $260 \mu \mathrm{m}$ ) compared to far-IR, submillimetre and radio observations of submillimetre galaxies (SMGs, marked by dots) at $1.4 \leqslant z \leqslant 5$ excluding SMGs with likely AGN. This plot indicates that for a carefully selected sample of high-redshift star-forming ULIRGs the radio-IR luminosity relation show no obvious deviation from that found locally.
350 and $850 \mu \mathrm{m}$. We have not shown the measurements of galaxies at $z<1.4$, and we also rejected measurements of three systems that are likely to be influenced by AGN and one more not detected at $350 \mu \mathrm{m}$ : numbers 2, 3,9 and 10 in their table 1 . Where they were available, we used the improved $1.4-\mathrm{GHz}$ radio flux densities measurements from Biggs \& Ivison (2006) instead of those from Kovács et al. (2006).

We normalized the plotted points to provide a good fit at both 350 and $850 \mu \mathrm{m}$ (observed) to the SEDs of local ULIRGs. As Fig. 1 shows, the radio and IR flux densities indicated for the high-redshift galaxies $(1.4 \leqslant z \leqslant 3.4)$ agree well with the envelope of the SEDs of the local ULIRGs. In the radio, there is no apparent offset from the local galaxies, but the scatter is considerably larger than for the local ULIRGs. However, a significant part of this larger scatter is likely due to measurement errors, since many of the $1.4-\mathrm{GHz}$ flux densities have relatively low $\mathrm{S} / \mathrm{N}$. A further explanation for the scatter is that the high-redshift starbursts have a wider range of radio spectral indices.

This paper has been typeset from a $\mathrm{T}_{\mathrm{E}} \mathrm{X} / \mathrm{L} \mathrm{T} \mathrm{T}_{\mathrm{E}} \mathrm{X}$ file prepared by the author. 